\title{
Possible realization of entanglement, logical gates, and quantum-information transfer with superconducting-quantum-interference-device qubits in cavity QED
}

\author{
Chui-Ping Yang* and Shih-I Chu ${ }^{\dagger}$ \\ Department of Chemistry, University of Kansas, Lawrence, Kansas 66045 \\ and Kansas Center for Advanced Scientific Computing, Lawrence, Kansas 66045 \\ Siyuan Han \\ Department of Physics and Astronomy, University of Kansas, Lawrence, Kansas 66045
}

(Received 9 October 2002; published 17 April 2003)

\begin{abstract}
We present a scheme to achieve maximally entangled states, controlled phase-shift gate, and SWAP gate for two superconducting-quantum-interference-device (SQUID) qubits, by placing SQUIDs in a microwave cavity. We also show how to transfer quantum information from one SQUID qubit to another. In this scheme, no transfer of quantum information between the SQUIDs and the cavity is required, the cavity field is only virtually excited and thus the requirement on the quality factor of the cavity is greatly relaxed.
\end{abstract}

DOI: 10.1103/PhysRevA.67.042311

PACS number(s): 03.67.Lx, 85.25.Dq, 89.70.+c, 42.50.Dv

\section{INTRODUCTION}

A number of groups have proposed how to perform quantum logic using superconducting devices such as Josephsonjunction circuits [1-3], Josephson junctions [4-7], Cooper pair boxes [8-12], and superconducting-quantuminterference devices (SQUIDs) [13-16]. These proposals play an important role in building up superconducting quantum computers. In this paper, we show a scheme for doing quantum logic with SQUID qubits in a microwave cavity. The proposal merges ideas from the quantum manipulation with atoms or ions in cavity QED [17-20]. The motivation for this scheme is fivefold given as the following

(i) About six years ago, SQUIDs were proposed as candidates to serve as the qubits for a superconducting quantum computer [21]. Recently, people have presented many methods for demonstrating macroscopic coherence of a SQUID $[22,23]$ or performing a single-"SQUID-qubit" logic operation [13-16], but did not give much report on how to achieve quantum logic for two SQUID qubits. As we know, the key ingredient in any quantum computation is the two-qubit gate. The present scheme shows a way to implement two-SQUIDqubit quantum logic gates.

(ii) Compared with the other noncavity SQUID-based schemes where significant resources may be involved in coupling two distant qubits, the present scheme may be simple as far as coupling qubits, since the cavity mode acts as a "bus" and can mediate long-distance, fast interaction between distant SQUID qubits.

(iii) SQUIDs are sensitive to environment. By placing SQUIDs into a superconducting cavity, decoherence induced due to the external environment can be greatly suppressed because the cavity can be doubled as the magnetic shield for SQUIDs.

\footnotetext{
*Email address: cpyang@floquet.chem.ku.edu

†Email address: sichu@ku.edu

‡Email address: han@ku.edu
}

(iv) It is known that certain kinds of atoms or ions have a weak coupling with environment and long decoherence time. Experiments have been made so far in the cavity-atom or cavity-ion systems, which demonstrated the feasibility of small-scale quantum computing. However, technically speaking, the cavity-SQUID scheme may be preferable for demonstration purposes to the cavity-atom or cavity-ion proposals, since SQUIDs can be easily embedded in a cavity, while the latter requires techniques for trapping atoms or ions.

(v) Quantum computation based on semiconductor quantum dots have been paid much interest, but recent reports show that superconducting devices have relatively long decoherence time $[24,25]$ compared with quantum dots [2630]. Decoherence time can reach the order of $1-5 \mu \mathrm{s}$ for superconducting devices [24,25]; while, for quantum dots, typical decoherence times for "the spin states of excess conduction electrons" and for "charge states of excitons" are, respectively, of the order of $100 \mathrm{~ns}$ [26-28] and the order of $1 \mathrm{~ns}$ [28-30]).

This paper focuses on quantum logical gates (the controlled phase-shift gate and the SWAP gate) of two SQUID qubits inside a cavity. The scheme does not require any transfer of quantum information between the SQUID system and the cavity, i.e., the cavity is only virtually excited. Thus, the cavity decay is suppressed during the gate operations. In addition, we discuss how to create maximally entangled states with two SQUID qubits and how to transfer quantum information from one SQUID qubit to another.

The paper is organized as follows. In Sec. II, we introduce the Hamiltonian of a SQUID coupled to a single-mode cavity field. In Sec. III, we consider a SQUID driven by a classical microwave pulse. In Sec. IV, we discuss how to achieve two-SQUID-qubit maximally entangled states, logical gates, and information transfer from one SQUID qubit to another. A brief discussion on the experimental issues and the summary are given in Sec. V.

\section{SQUID COUPLED TO CAVITY FIELD}

Consider a system composed of a SQUID coupled to a single-mode cavity field (assuming that all other cavity 
modes are well decoupled to the three energy levels of the SQUID). The Hamiltonian of the coupled system $H$ can be written as a sum of the energies of the cavity field and the SQUID, plus a term for the interaction energy,

$$
H=H_{c}+H_{s}+H_{I},
$$

where $H_{c}, H_{s}$, and $H_{I}$ are the Hamiltonian of the cavity field, the Hamiltonian of the SQUID, and the interaction energy, respectively.

The SQUIDs considered throughout this paper are rf SQUIDs, each consisting of Josephson tunnel junction enclosed by a superconducting loop (the size of an rf SQUID is on the order of 10-100 $\mu \mathrm{m}$ ). The Hamiltonian for an $\mathrm{rf}$ SQUID (with junction capacitance $C$ and loop inductance $L$ ) can be written in the usual form [31,32]

$$
H_{s}=\frac{Q^{2}}{2 C}+\frac{\left(\Phi-\Phi_{x}\right)^{2}}{2 L}-E_{J} \cos \left(2 \pi \frac{\Phi}{\Phi_{0}}\right),
$$

where $\Phi$ that is the magnetic flux threading the ring and $Q$ that is the total charge on the capacitor are the conjugate variables of the system (with the commutation relation $[\Phi, Q]=i \hbar), \Phi_{x}$ is the static (or quasistatic) external flux applied to the ring, and $E_{J} \equiv I_{c} \Phi_{0} / 2 \pi$ is the Josephson coupling energy $\left(I_{c}\right.$ is the critical current of the junction and $\Phi_{0}=h / 2 e$ is the flux quantum).

The Hamiltonian of the single-mode cavity field can be written as

$$
H_{c}=\hbar \omega_{c}\left(a^{\dagger} a+\frac{1}{2}\right)
$$

where $a^{\dagger}$ and $a$ are the creation and annihilation operators of the cavity field; and $\omega_{c}$ is the frequency of the cavity field.

The cavity field and the SQUID ring are coupled together inductively with a coupling energy given by

$$
H_{I}=\lambda_{c}\left(\Phi-\Phi_{x}\right) \Phi_{c},
$$

where $\lambda_{c}=-1 / L$ is the coupling parameter linking the cavity field to the SQUID ring; and $\Phi_{c}$ is the magnetic flux threading the ring, which is generated by the magnetic component $\mathbf{B}(\mathbf{r}, t)$ of the cavity field. The expression of $\Phi_{c}$ is given by

$$
\Phi_{c}=\int_{S} \mathbf{B}(\mathbf{r}, t) \cdot d \mathbf{S}
$$

( $S$ is any surface that is bounded by the ring, and $\mathbf{r}$ is the position vector of a point on $S)$. $\mathbf{B}(\mathbf{r}, t)$ takes the following form:

$$
\mathbf{B}(\mathbf{r}, t)=\sqrt{\frac{\hbar \omega_{c}}{2 \mu_{0}}}\left[a(t)+a^{\dagger}(t)\right] \mathbf{B}(\mathbf{r}),
$$

where $\mathbf{B}(\mathbf{r})$ is the magnetic component of the normal mode of the cavity.

We denote $|n\rangle$ as the ( $\Phi_{x}$ dependent) eigenstate of $H_{s}$ with an eigenvalue $E_{n}$. Based on the completeness relation $\Sigma_{n}|n\rangle\langle n|=I$, it follows from Eqs. (2) and (4) that

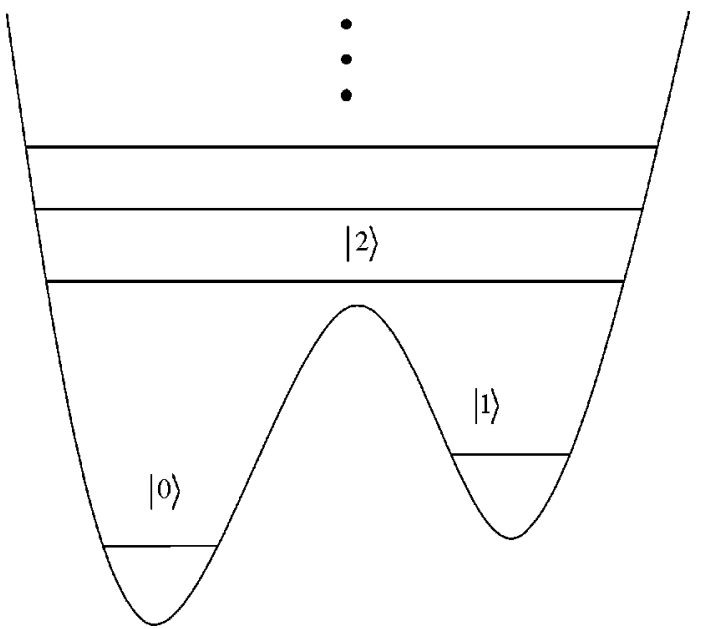

FIG. 1. Level diagram of a SQUID with the $\Lambda$-type three lowest levels $|0\rangle,|1\rangle$, and $|2\rangle$.

$$
\begin{gathered}
H_{s}=\sum_{n} E_{n}|n\rangle\langle n|, \\
H_{I}=\sum_{n}|n\rangle\left\langle n\left|H_{I} \sum_{m}\right| m\right\rangle\langle m| \\
=\lambda_{c} \Phi_{c} \sum_{n, m}|n\rangle\left\langle n\left|\Phi-\Phi_{x}\right| m\right\rangle\langle m| .
\end{gathered}
$$

Let us consider the $\Lambda$-type three lowest levels of a SQUID, denoted by $|0\rangle,|1\rangle$, and $|2\rangle$, respectively (shown in Fig. 1). If the coupling of $|0\rangle,|1\rangle$ and $|2\rangle$ with other levels via cavity modes is negligible, we have (setting $\hbar=1$ below)

$$
H_{s}=E_{0}|0\rangle\left\langle 0\left|+E_{1}\right| 1\right\rangle\left\langle 1\left|+E_{2}\right| 2\right\rangle\langle 2|
$$

and

$$
\begin{aligned}
H_{I}= & \left(a+a^{\dagger}\right)\left(g_{00}|0\rangle\left\langle 0\left|+g_{11}\right| 1\right\rangle\left\langle 1\left|+g_{22}\right| 2\right\rangle\langle 2|\right) \\
& +g_{01} a|0\rangle\left\langle 1\left|+g_{12} a\right| 1\right\rangle\left\langle 2\left|+g_{02} a\right| 0\right\rangle\left\langle 2\left|+g_{10} a^{\dagger}\right| 1\right\rangle\langle 0| \\
& +g_{21} a^{\dagger}|2\rangle\left\langle 1\left|+g_{20} a^{\dagger}\right| 2\right\rangle\left\langle 0\left|+g_{01} a^{\dagger}\right| 0\right\rangle\langle 1| \\
& +g_{12} a^{\dagger}|1\rangle\left\langle 2\left|+g_{02} a^{\dagger}\right| 0\right\rangle\left\langle 2\left|+g_{10} a\right| 1\right\rangle\langle 0| \\
& +g_{21} a|2\rangle\left\langle 1\left|+g_{20} a\right| 2\right\rangle\langle 0|,
\end{aligned}
$$

where

$$
\begin{aligned}
& g_{i i}=\lambda_{c} \sqrt{\left(\hbar \omega_{c} / 2 \mu_{0}\right)}\left(\langle i|\Phi| i\rangle-\Phi_{x}\right) \widetilde{\Phi}_{c}, \\
& g_{i j}=\lambda_{c} \sqrt{\left(\hbar \omega_{c} / 2 \mu_{0}\right)}\langle i|\Phi| j\rangle \widetilde{\Phi}_{c}
\end{aligned}
$$

[here, $\widetilde{\Phi}_{c}=\int_{S} \mathbf{B}(\mathbf{r}) \cdot d \mathbf{S} ; i, j=0,1,2$, and $\left.i \neq j\right]$. For simplicity, we will choose $g_{i j}=g_{j i}$ since eigenfunctions of $H_{s}$ can in general be chosen to be real.

In the case where the cavity field is far-off resonant with the transition between the levels $|0\rangle$ and $|1\rangle$ as well as the transition between the levels $|1\rangle$ and $|2\rangle$, the Hamiltonian (9) reduces to 


$$
\begin{aligned}
H_{I}= & \left(a+a^{\dagger}\right)\left(g_{00}|0\rangle\left\langle 0\left|+g_{11}\right| 1\right\rangle\left\langle 1\left|+g_{22}\right| 2\right\rangle\langle 2|\right) \\
& +g_{02} a|0\rangle\left\langle 2\left|+g_{20} a^{\dagger}\right| 2\right\rangle\left\langle 0\left|+g_{02} a^{\dagger}\right| 0\right\rangle\left\langle 2\left|+g_{20} a\right| 2\right\rangle\langle 0| .
\end{aligned}
$$

It follows from Eqs. (3), (8), and (10) that the interaction Hamiltonian in the interaction picture is given by

$$
\begin{aligned}
H_{I}= & \left(e^{-i \omega_{c} t} a+e^{i \omega_{c} t} a^{\dagger}\right)\left(g_{00}|0\rangle\left\langle 0\left|+g_{11}\right| 1\right\rangle\left\langle 1\left|+g_{22}\right| 2\right\rangle\langle 2|\right) \\
& +g_{02} e^{-i\left(\omega_{c}+\omega_{20}\right) t} a|0\rangle\left\langle 2\left|+g_{20} e^{i\left(\omega_{c}+\omega_{20}\right) t} a^{\dagger}\right| 2\right\rangle\langle 0| \\
& +g_{02} e^{i\left(\omega_{c}-\omega_{20}\right) t} a^{\dagger}|0\rangle\left\langle 2\left|+g_{20} e^{-i\left(\omega_{c}-\omega_{20}\right) t} a\right| 2\right\rangle\langle 0|,
\end{aligned}
$$

where $\omega_{20} \equiv\left(E_{2}-E_{0}\right) / \hbar$ is the transition frequency between the levels $|0\rangle$ and $|2\rangle$.

From Eq. (11) one can see that if the following condition is satisfied:

$$
\omega_{c} \gg \Delta=\omega_{c}-\omega_{20},
$$

i.e., the cavity-field frequency is much larger than the detuning from the transition frequency between the levels $|0\rangle$ and $|2\rangle$, we can discard the rapidly oscillating terms in the Hamiltonian (11) (i.e., the rotating-wave approximation). Thus, the final effective interaction Hamiltonian (in the interaction picture) has the form

$$
H_{I}=g_{02}\left[e^{i\left(\omega-\omega_{20}\right) t} a^{\dagger}|0\rangle\left\langle 2\left|+e^{-i\left(\omega-\omega_{20}\right) t} a\right| 2\right\rangle\langle 0|\right],
$$

where $g_{02}$ is the coupling constant between the SQUID and the cavity field, corresponding to the transitions between $|0\rangle$ and $|2\rangle$.

\section{SQUID DRIVEN BY A MICROWAVE PULSE}

Now, let us consider a SQUID driven by a classical microwave pulse (without cavity). In the following, the SQUID is still treated quantum mechanically, while the microwave pulse is treated classically. The Hamiltonian $H$ for the coupled system can be written as

$$
H=H_{s}+H_{I},
$$

where $H_{s}$ and $H_{I}$ are the Hamiltonians (2) for the SQUID and the interaction energy (between the SQUID and the microwave pulse), respectively. The expression of $H_{I}$ is given by

$$
H_{I}=\lambda_{\mu w}\left(\Phi-\Phi_{x}\right) \Phi_{\mu w},
$$

where $\lambda_{\mu w}=-1 / L$ is a coupling coefficient linking the microwave field to the SQUID ring; $\Phi_{\mu w}$ is the magnetic flux threading the ring, which is generated by the magnetic component $\mathbf{B}^{\prime}(\mathbf{r}, t)=\mathbf{B}^{\prime}(\mathbf{r}) \cos \omega_{\mu w} t$ of the microwave pulse, and has the following form:

$$
\Phi_{\mu w}=\int_{S} \mathbf{B}^{\prime}(\mathbf{r}, t) \cdot d \mathbf{S} \equiv \widetilde{\Phi}_{\mu w} \cos \omega_{\mu w} t
$$

[here, $\widetilde{\Phi}_{\mu w}=\int_{S} \mathbf{B}^{\prime}(\mathbf{r}) \cdot d \mathbf{S}$, the notations of $S$ and $\mathbf{r}$ are the same as described before, and $\omega_{\mu w}$ is the frequency of the microwave pulse]. Assume that the microwave pulse is resonant with the transition between the levels $|0\rangle$ and $|2\rangle$. Using the above procedures, the interaction Hamiltonian in the interaction picture is then

$$
\begin{aligned}
H_{I}= & \Omega_{00}\left(e^{i \omega_{\mu w} t}+e^{-i \omega_{\mu w}}\right)|0\rangle\left\langle 0\left|+\Omega_{22}\left(e^{i \omega_{\mu w} t}+e^{-i \omega_{\mu w}}\right)\right| 2\right\rangle \\
& \times\left\langle 2\left|+\Omega_{02}\left[e^{-i\left(\omega_{\mu w}+\omega_{20}\right) t}+e^{i\left(\omega_{\mu w}-\omega_{20}\right) t}\right]\right| 0\right\rangle\langle 2| \\
& +\Omega_{20}\left[e^{i\left(\omega_{\mu w}+\omega_{20}\right) t}+e^{-i\left(\omega_{\mu w}-\omega_{20}\right) t}\right]|2\rangle\langle 0|,
\end{aligned}
$$

where $\quad \Omega_{i i}=\lambda_{\mu w}\left(\langle i|\Phi| i\rangle-\Phi_{x}\right) \widetilde{\Phi}_{\mu w}$, $=\lambda_{\mu w}\langle i|\Phi| j\rangle \Phi_{\mu w}$, and $\Omega_{i j}=\Omega_{j i}(i, j=0,2$ and $i \neq j)$. In the case of resonance $\left(\omega_{\mu w}=\omega_{20}\right)$ and under the rotatingwave approximation, the interaction Hamiltonian (17) reduces to

$$
H_{I}=\Omega_{02}(|0\rangle\langle 2|+| 2\rangle\langle 0|)
$$

where $\Omega_{02}$ is the frequency of the Rabi oscillation between the levels $|0\rangle$ and $|2\rangle$. Based on Eq. (18), it is easy to get the following state rotation

$$
\begin{gathered}
|0\rangle \rightarrow \cos \Omega_{02} t|0\rangle-i \sin \Omega_{02} t|2\rangle, \\
|2\rangle \rightarrow-i \sin \Omega_{02} t|0\rangle+\cos \Omega_{02} t|2\rangle .
\end{gathered}
$$

Similarly, when the microwave pulse frequency is tuned with the transition frequency $\omega_{21} \equiv\left(E_{2}-E_{1}\right) / \hbar$ between the levels $|1\rangle$ and $|2\rangle$, we have

$$
H_{I}=\Omega_{12}(|1\rangle\langle 2|+| 2\rangle\langle 1|) .
$$

Comparing $|1\rangle$ and $|2\rangle$ of Eq. (20) with $|0\rangle$ and $|2\rangle$ of Eq. (18), respectively, it is clear that we have

$$
\begin{gathered}
|1\rangle \rightarrow \cos \Omega_{12} t|1\rangle-i \sin \Omega_{12} t|2\rangle, \\
|2\rangle \rightarrow-i \sin \Omega_{12} t|1\rangle+\cos \Omega_{12} t|2\rangle,
\end{gathered}
$$

where $\Omega_{12}=\lambda_{\mu w}\langle 1|\Phi| 2\rangle \widetilde{\Phi}_{\mu w}$ is the Rabi frequency between the levels $|1\rangle$ and $|2\rangle$.

Finally, for the two-dimensional Hilbert space made of $|0\rangle$ and $|1\rangle$, an arbitrary rotation

$$
\begin{gathered}
|0\rangle \rightarrow \cos \Omega_{01} t|0\rangle-i \sin \Omega_{01} t|1\rangle, \\
|1\rangle \rightarrow-i \sin \Omega_{01} t|0\rangle+\cos \Omega_{01} t|1\rangle
\end{gathered}
$$

(where $\Omega_{01}=\lambda_{\mu w}\langle 0|\Phi| 1\rangle \widetilde{\Phi}_{\mu w}$ ) can be implemented if the microwave frequency is tuned with the transition frequency $\omega_{10} \equiv\left(E_{1}-E_{0}\right) / \hbar$ between the levels $|0\rangle$ and $|1\rangle$. In the following discussions, this rotation will not be employed, since it requires very long gate time due to the barrier between the levels $|0\rangle$ and $|1\rangle[15]$. 

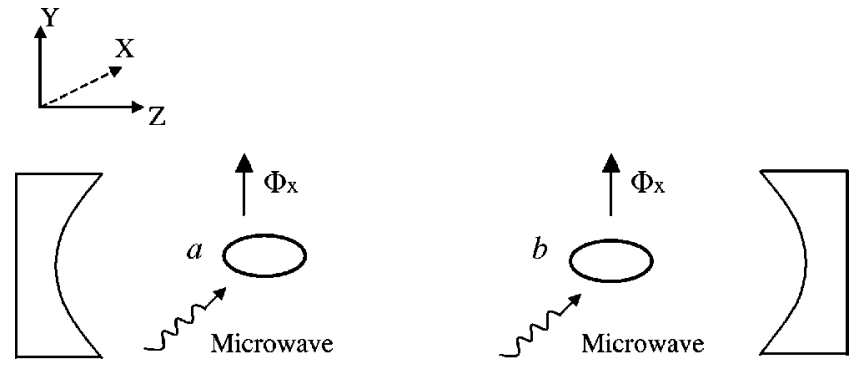

FIG. 2. Schematic illustration of two SQUIDs $(a, b)$ coupled to a single-mode cavity field and manipulated by microwave pulses. The two SQUIDs are placed along the cavity axis (i.e., the $Z$ axis). The microwave pulses propagate in the $X-Z$ plane (parallel to the surface of the SQUID ring), with the magnetic-field component perpendicular to the surface of the SQUID ring.

\section{ENTANGLEMENT, LOGICAL GATE, AND INFORMATION TRANSFER}

In this section, we consider two identical SQUIDs $a$ and $b$ coupled to a single-mode microwave cavity (Fig. 2). The separation of the two SQUIDs is assumed to be much larger than the linear dimension of each SQUID ring in such a way that the interaction between the two SQUIDs is negligible. Also, assume that the coupling of each SQUID to the cavity field is the same [this can be readily obtained by setting the two SQUIDs on two different places $\mathbf{r}_{1}$ and $\mathbf{r}_{2}$ of the cavity axis where the cavity-field magnetic components $\mathbf{B}\left(\mathbf{r}_{1}, t\right)$ and $\mathbf{B}\left(\mathbf{r}_{2}, t\right)$ are the same]. If the above assumption applies, i.e., for each SQUID the coupling of the three lowest levels $|0\rangle,|1\rangle$, and $|2\rangle$ with other levels via cavity modes is negligible and the cavity field is far-off resonant with the transition between the levels $|0\rangle$ and $|1\rangle$ as well as the transition between the levels $|1\rangle$ and $|2\rangle$, it is obvious that based on Eq. (13), the interaction Hamiltonian between the two SQUIDs and the cavity field in the interaction picture can be written as

$$
\begin{aligned}
H_{I}= & g_{02} \sum_{m=a, b}\left(e^{-i\left(\omega_{c}-\omega_{20}\right) t} a|2\rangle_{m}\langle 0|\right. \\
& \left.+e^{i\left(\omega_{c}-\omega_{20}\right) t} a^{\dagger}|0\rangle_{m}\langle 2|\right),
\end{aligned}
$$

where the subscript $m$ represents SQUID $a$ or $b$. In the case of $\omega_{c}-\omega_{20} \gg g_{02}$, i.e., the detuning between the transition frequency (for the levels $|0\rangle$ and $|2\rangle$ ) and the cavity-field frequency is much larger than the corresponding coupling constant, there is no energy exchange between the SQUIDs and the cavity field. The effective Hamiltonian is then given by $[33,34]$

$$
\begin{aligned}
H= & \gamma\left[\sum_{m=a, b}\left(|2\rangle_{m}\left\langle 2\left|a a^{\dagger}-\right| 0\right\rangle_{m}\langle 0| a^{\dagger} a\right)\right. \\
& \left.+|2\rangle_{a}\langle 0|\otimes| 0\rangle_{b}\langle 2|+| 0\rangle_{a}\langle 2|\otimes| 2\rangle_{b}\langle 0|\right],
\end{aligned}
$$

where $\gamma=g_{02}^{2} /\left(\omega-\omega_{20}\right)$. The first and second terms of Eq. (24) describe the photon-number dependent Stark shifts, while the third and fourth terms describe the "dipole" cou- pling between the two SQUIDs mediated by the cavity mode. If the cavity field is initially in the vacuum state, the Hamiltonian (24) reduces to

$$
\begin{aligned}
H= & \gamma\left[\sum_{m=a, b}|2\rangle_{m}\langle 2|+| 2\rangle_{a}\langle 0|\otimes| 0\rangle_{b}\langle 2|\right. \\
& \left.+|0\rangle_{a}\langle 2|\otimes| 2\rangle_{b}\langle 0|\right] .
\end{aligned}
$$

Note that the Hamiltonian (25) does not contain the operators of the cavity field. Thus, only the state of the SQUID system undergoes an evolution under the Hamiltonian (25), i.e., no quantum-information transfer exists between the SQUID system and the cavity field. Therefore, the cavity field is virtually excited.

It is clear that the states $|0\rangle_{a}|0\rangle_{b}$ and $|0\rangle_{a}|1\rangle_{b}$ are unaffected under the Hamiltonian (25) during the SQUID-cavity interaction. From Eq. (25), one can easily get the following state evolution:

$$
\begin{aligned}
& |2\rangle_{a}|0\rangle_{b} \rightarrow e^{-i \gamma t}\left[\cos (\gamma t)|2\rangle_{a}|0\rangle_{b}-i \sin (\gamma t)|0\rangle_{a}|2\rangle_{b}\right] \\
& |0\rangle_{a}|2\rangle_{b} \rightarrow e^{-i \gamma t}\left[\cos (\gamma t)|0\rangle_{a}|2\rangle_{b}-i \sin (\gamma t)|2\rangle_{a}|0\rangle_{b}\right] \\
& |2\rangle_{a}|2\rangle_{b} \rightarrow e^{-i 2 \gamma t}|2\rangle_{a}|2\rangle_{b} \\
& |2\rangle_{a}|1\rangle_{b} \rightarrow e^{-i \gamma t}|2\rangle_{a}|1\rangle_{b} .
\end{aligned}
$$

In the following, we will show that Eq. (26) can be used to create entanglement to perform logical gates and to implement quantum-information transfer.

The operations described in the rest of this paper can be realized by means of the following three-step state manipulation.

(i) First, adjust the level spacing of each SQUID so that the transition between any two levels is far-off resonant with the cavity field [in this case, the interaction between the SQUIDs and the cavity field is turned off since the interaction Hamiltonian (25) $H \approx 0$ ].

(ii) Apply a resonant microwave pulse to one of the SQUIDs so that the state of this SQUID undergoes a transformation.

(iii) Finally, adjust the level spacing of each SQUID back to the original configuration, i.e., only the transitions $|1\rangle \leftrightarrow$ $|2\rangle$ and $|0\rangle \leftrightarrow|1\rangle$ are far-off resonant with the cavity field so that the system will undergo an evolution under the Hamiltonian (25). In the SQUID system, the level spacing can be easily changed by adjusting the external flux $\Phi_{x}$ or the critical current $I_{c}$ (for variable barrier rf SQUIDs). To simplify our discussion, we call this three-step process "ARA" (shown in Fig. 3).

\section{A. Generation of entanglement}

Entanglement is considered to be one of the most profound features of quantum mechanics. An entangled state of a system consisting of two subsystems cannot be described as a product of the quantum states of the two subsystems. In this sense, the entangled system is considered inseparable 


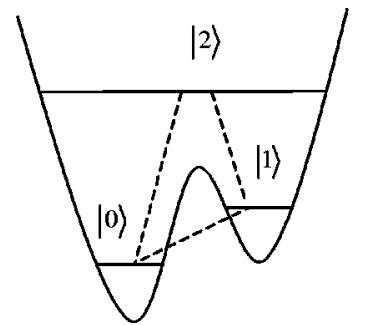

(a)

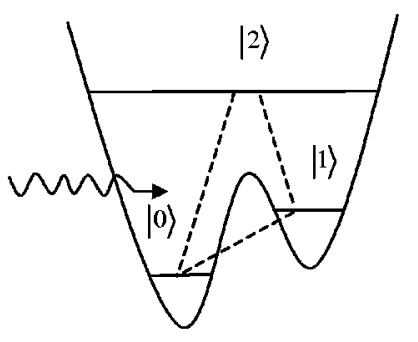

(b)

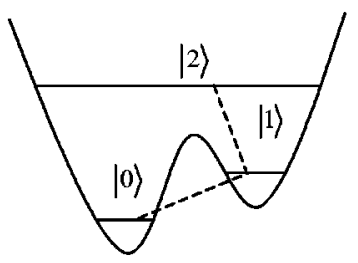

(c)

FIG. 3. Illustration of the ARA process. (a) The reduced level structure for each SQUID after adjusting the level spacings; (b) a microwave pulse with $\omega_{\mu w} \equiv \omega_{20}$ or $\omega_{21}$ being applied to the SQUID $a$ or the SQUID $b$; (c) the reduced level structure for each SQUID after adjusting the level spacings back to that of before step (a). Transitions between levels linked by dashed lines are far-off resonant with the cavity field.

[35]. Recently, there has been much interest in practical applications of entangled states in quantum computation, quantum cryptography, quantum teleportation, and so on [36-39]. Experimental realizations of entangled states with up to four photons [40], up to four trapped ions [41], or two atoms in microwave cavity QED [42] have been reported.

Assume that two SQUIDs are initially in the states $|0\rangle_{a}$ and $|0\rangle_{b}$. In order to prepare the two SQUID qubits in the maximally entangled state, we apply an ARA process in which a $\pi$ - microwave pulse $\left(2 \Omega_{02} t=\pi\right.$, where $t$ is the pulse duration), resonant with the transition $|0\rangle_{a} \leftrightarrow|2\rangle_{a}$, is applied to the SQUID $a$. In this way, we obtain the transformation $|0\rangle_{a} \rightarrow-i|2\rangle_{a}$, i.e., the state $|0\rangle_{a}|0\rangle_{b}$ becomes $-i|2\rangle_{a}|0\rangle_{b}$. After this ARA process, let the state of the SQUID system evolve under the Hamiltonian (25). From Eq. (26), one can see that after an interaction time $\pi /(4 \gamma)$, the two SQUIDs will be in the maximally entangled state

$$
|\psi\rangle=-\frac{1}{\sqrt{2}}\left(|0\rangle_{a}|2\rangle_{b}+i|2\rangle_{a}|0\rangle_{b}\right)
$$

where the common phase factor $e^{-i \pi / 4}$ has been omitted. Note that the rate of energy relaxation of level $|1\rangle$ is much smaller than that of level $|2\rangle$ because of the barrier between the levels $|0\rangle$ and $|1\rangle$ of the SQUIDs. Hence, to reduce decoherence, the state (27) is transformed into

$$
|\psi\rangle=\frac{1}{\sqrt{2}}\left(i|0\rangle_{a}|1\rangle_{b}-|1\rangle_{a}|0\rangle_{b}\right)
$$

by applying a second ARA process, in which each SQUID interacts with a $\pi$ microwave pulse (resonant with $\omega_{21}$ ), resulting in the transformation $|2\rangle \rightarrow-i|1\rangle$ for each SQUID. The prepared state (28) is a maximally entangled state of two SQUID qubits $a$ and $b$ (here and in the following, the two orthogonal states of a SQUID qubit are denoted by the two lowest-energy states $|0\rangle$ and $|1\rangle$ ).

\section{B. Controlled phase-shift gate}

Assume that SQUID qubit $a$ is a control bit and SQUID qubit $b$ is a target bit. The controlled phase-shift (CPS) gate can be realized in the following three steps:

Step (i) Apply an ARA process in which a $\pi$ pulse with $\omega_{\mu w}=\omega_{21}$ is applied to SQUID $a$, resulting in the transformation $|1\rangle_{a} \rightarrow-i|2\rangle_{a}$.

Step (ii) After the ARA process, let the state of the two SQUIDs undergo an evolution for an interaction time $\pi / \gamma$ under the Hamiltonian (25).

Step (iii) Apply an ARA process again in which a $3 \pi$ pulse with $\omega_{\mu w}=\omega_{21}$ is applied to SQUID $a$, resulting in the transformation $|2\rangle_{a} \rightarrow i|1\rangle_{a}$.

The states of the two-SQUID system after each step of the three transformations are summarized in the following table:

$$
\begin{aligned}
& |0\rangle_{a}|0\rangle_{b} \\
& |0\rangle_{a}|0\rangle_{b} \\
& |0\rangle_{a}|1\rangle_{b} \stackrel{\text { Step (i) }}{\longrightarrow} \\
& |0\rangle_{a}|1\rangle_{b} \\
& |1\rangle_{a}|0\rangle_{b} \\
& -i|2\rangle_{a}|0\rangle_{b} \\
& |1\rangle_{a}|1\rangle_{b} \\
& -i|2\rangle_{a}|1\rangle_{b} \\
& |0\rangle_{a}|0\rangle_{b} \quad|1\rangle_{a}|1\rangle_{b} \\
& |0\rangle_{a}|1\rangle_{b} \stackrel{\text { Step (iii) }}{\longrightarrow}|0\rangle_{a}|1\rangle_{b} \\
& -i|2\rangle_{a}|0\rangle_{b} \quad|1\rangle_{a}|0\rangle_{b} \\
& i|2\rangle_{a}|1\rangle_{b} \quad-|1\rangle_{a}|1\rangle_{b},
\end{aligned}
$$

which shows that a universal two SQUID-qubit CPS gate is realized.

A two-qubit controlled-NOT (CNOT) gate can be obtained by combining a two-qubit CPS gate with two single-qubit rotation gates [43]. Thus, applying the ARA procedures to implement single-SQUID qubit rotating operations, together with the above CPS gate operations is sufficient to obtain the two-SQUID qubit controlled-NOT gate.

\section{SWAP gate}

It is known that construction of a SWAP gate requires at least three controlled-NOT gates as follows [44]: 


$$
\begin{aligned}
|i\rangle_{a}|j\rangle_{b} & \rightarrow|i\rangle_{a}|i \oplus j\rangle_{b} \\
& \rightarrow|i \oplus(i \oplus j)\rangle_{a}|i \oplus j\rangle_{b}=|j\rangle_{a}|i \oplus j\rangle_{b} \\
& \rightarrow|j\rangle_{a}|(i \oplus j) \oplus j\rangle_{b}=|j\rangle_{a}|i\rangle_{b},
\end{aligned}
$$

where $i, j \in\{0,1\}$ and all additions are done modulo 2. As described above, a CNOT can be realized with a CPS and two single-qubit rotations. Since each two-SQUID-qubit CPS gate requires three basic steps described above, at least nine basic steps for three CPS gates, together with six singleSQUID-qubit rotation operations are needed to implement a two-SQUID-qubit SWAP gate by using the above method. In the discussion below, we present a different way to perform a SWAP, which requires only the following five steps.

Step (i): Apply an ARA process in which each SQUID
$|0\rangle_{a}|0\rangle_{b}$
$|0\rangle_{a}|0\rangle_{b}$
$|0\rangle_{a}|0\rangle_{b}$
$|0\rangle_{a}|1\rangle_{b} \stackrel{\text { Step (i) }}{\longrightarrow}-i|0\rangle_{a}|2\rangle_{b} \stackrel{\text { Step (ii) }}{\longrightarrow}$
$i|2\rangle_{a}|0\rangle_{b} \stackrel{\text { Step (iii) }}{\longrightarrow}$
$|1\rangle_{a}|0\rangle_{b}$
$-i|2\rangle_{a}|0\rangle_{b}$
$i|0\rangle_{a}|2\rangle_{b}$
$|1\rangle_{a}|1\rangle_{b}$
$-|2\rangle_{a}|2\rangle_{b}$
$|2\rangle_{a}|2\rangle_{b}$

$|0\rangle_{a}|0\rangle_{b}$

$|0\rangle_{a}|0\rangle_{b}$

$|0\rangle_{a}|0\rangle_{b}$ interacts with a $\pi$ pulse (resonant with $\omega_{21}$ ) so that each SQUID undergoes the transformation $|1\rangle \rightarrow-i|2\rangle$.

Step (ii): Let the state of the SQUID system undergo an evolution for an interaction time $\pi /(2 \gamma)$ under the Hamiltonian (25).

Step (iii): Perform an ARA process in which a $2 \pi$ pulse and a $\pi$ pulse, resonant with $\omega_{21}$ of the SQUID $a$ and the SQUID $b$, respectively, are applied, resulting in transformations $|2\rangle_{a} \rightarrow-|2\rangle_{a}$ and $|2\rangle_{b} \rightarrow-i|1\rangle_{b}$.

Step (iv): Let the state of the system undergo an evolution for an interaction time $\pi / \gamma$ under the Hamiltonian (25).

Step (v): Perform an ARA process in which a $3 \pi$ pulse, resonant with $\omega_{21}$, is applied to the SQUID $a$ so that it undergoes the transformation $|2\rangle_{a} \rightarrow i|1\rangle_{a}$.

The states after each step of the above operations are listed below:
It is clear that the operations accomplish a two-SQUID-qubit SWAP gate.

\section{Transfer of information}

Recently, quantum teleportation [38] has been paid much interest because it plays an important role in quantuminformation processing. It is also noted that short-distance quantum teleportation can be applied to transport quantum information inside a quantum computer [45]. It is well known that transferring quantum information from one qubit to another requires a minimum number of three qubits by using the standard teleportation protocols $[38,45]$. In the following, we will present a different approach for transferring quantum information from one SQUID qubit to another by the use of only two SQUID qubits.

Assume that the SQUID qubit $a$ is the original carrier of quantum information, which is in an arbitrary state $\alpha|0\rangle$ $+\beta|1\rangle$; and we want to transfer this state from SQUID qubit $a$ to SQUID qubit $b$. To do this, the SQUID qubit $b$ is first prepared in the state $|0\rangle$. The quantum-state transfer between the two SQUID qubits is described by

$$
\left(\alpha|0\rangle_{a}+\beta|1\rangle_{a}\right)|0\rangle_{b} \rightarrow|0\rangle_{a}\left(\alpha|0\rangle_{b}+\beta|1\rangle_{b}\right) .
$$

From Eq. (32) one can see that this process can be done via a transformation that satisfies the following truth table:

$$
\begin{gathered}
|0\rangle_{a}|0\rangle_{b} \rightarrow|0\rangle_{a}|0\rangle_{b}, \\
|1\rangle_{a}|0\rangle_{b} \rightarrow|0\rangle_{a}|1\rangle_{b},
\end{gathered}
$$

which can be realized in the following three steps.

Step (i): Perform an ARA process in which a $\pi$ pulse $\left(\omega_{\mu w}=\omega_{21}\right)$ is applied to the SQUID $a$, resulting in the transformation $|1\rangle_{a} \rightarrow-i|2\rangle_{a}$.

Step (ii): Let the state of the two SQUIDs undergo an evolution for an interaction time $\pi /(2 \gamma)$ under the Hamiltonian (25).

Step (iii): Perform an ARA process in which a $\pi$ pulse $\left(\omega_{\mu w}=\omega_{21}\right)$ is applied to the SQUID $b$, resulting in the transformation $|2\rangle_{b} \rightarrow-i|1\rangle_{b}$. low:

The truth table of the entire operation is summarized be- 
It is easy to verify that the operations described above achieve the desired two-SQUID-qubit teleportation (32).

From above descriptions, one can also see that in each ARA process, no simultaneous $|0\rangle \rightarrow|2\rangle$ and $|1\rangle \rightarrow|2\rangle$ transitions are required for each SQUID and hence it is unnecessary to have the microwave pulses applied to two SQUIDs at the same time. Thus, it is sufficient to use only one microwave source with fixed frequency $\omega_{\mu w}$, since the transition frequency $\omega_{20}$ and $\omega_{21}$ of each SQUID can be rapidly adjusted to meet the resonant condition $\left(\omega_{\mu w}=\omega_{i j}\right)$, and the microwave can be redirected from one SQUID to another.

\section{DISCUSSION AND CONCLUSION}

Some experimental matters may need to be addressed here. First, the required time $t_{o p}$ for any gate operation (SWAP, CPS, CNOT, etc.) should be shorter than the energy relaxation time $t_{r}$ of level $|2\rangle$. The lifetime of the cavity mode is given by $T_{c}=Q / 2 \pi \nu$, where $Q$ is the quality factor of the cavity and $\nu$ is the cavity-field frequency. In our scheme, the cavity has a probability $P \simeq t_{o p} / t_{r}$ of being excited during the operation. Thus, the effective decay time of the cavity is $T_{c} / P$, which should be larger than the energy relaxation time $t_{r}$, i.e., the quality factor of the cavity should satisfy $Q \gg 2 \pi \nu t_{o p}$. The SQUIDs can be designed so that the level $|2\rangle$ has a sufficiently long energy relaxation time and thus the spontaneous decay of the SQUIDs is negligible during the operation. On the other hand, we can also use a high- $Q$ cavity and reduce the operation time by increasing the intensity of the microwave pulses and/or the coupling constant $g_{02}$ (e.g., by varying the energy-level structure of the SQUIDs) so that the cavity dissipation is negligible during the operation.

For the sake of definitiveness, let us consider the SQUIDs described in Ref. [15] for which the energy relaxation time $t_{r}$ of the level $|2\rangle$ could exceed $1 \mu$ s [24], the transition frequency $\nu_{0}$ between $|0\rangle$ and $|2\rangle$ is of the order of $80 \mathrm{GHz}$, and the typical gate time is $t_{o p} \simeq 0.01 t_{r}$. Taking $t_{r}=1 \mu \mathrm{s}$, $\nu_{0}=80 \mathrm{GHz}$ and the detuning $\nu-\nu_{0}=0.1 \mathrm{GHz}$, a simple calculation shows that the quality factor of the required cavity should be greater than $5 \times 10^{3}$, which is readily available in most laboratories. For instance, a superconducting cavity with a quality factor $Q=10^{8}$ has been demonstrated by Brune et al. [46].

It can be seen that the key element of the scheme is the ARA process. As discussed previously, the realization of ARA process requires rapid adjustments of level spacings of SQUIDs. The applied microwave pulses are ensured to be far-off resonant with the cavity field during each ARA process because $\omega_{20}$ and $\omega_{21}$ are highly detuned from $\omega_{c}$. Thus, the use of the microwave pulses does not change photon population in the cavity field. The scheme presented here has the following advantages. (i) using only two SQUID qubits (teleportation); (ii) faster (using three-level gates) [15]; (iii) not requiring very high- $Q$ microwave cavity; (iv) no need of

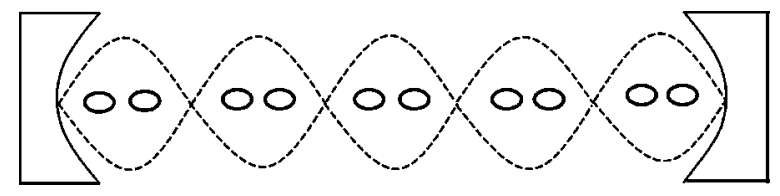

FIG. 4. Setup for quantum computing with many SQUIDs in a cavity. The interaction between any two SQUIDs is mediated through a single-mode standing-wave cavity field. During a logical gate operation on any two chosen SQUIDs, all other SQUIDs can be decoupled by adjusting the level spacings so that the transition between any two levels of each other SQUID is far-off resonant with the cavity field.

changing the microwave frequency $\omega_{\mu w}$ during the entire operation for all of the gates described; (v) possibility of being extended to perform quantum computing on lots of SQUID qubits inside a cavity (shown in Fig. 4) due to longdistance coherent interaction between SQUID qubits mediated via the cavity mode.

In summary, we have proposed a different scheme to create two-SQUID-qubit maximally entangled state and to implement two-SQUID-qubit logical gates (SWAP, CPS, and CNOT) with the use of a microwave cavity. The method can also be used to realize information transfer from one to another SQUID qubit (local teleportation) with two, instead of three qubits. The method does not require the transfer of quantum information between the cavity and the SQUID system. The cavity is only virtually excited during the whole operation; thus the requirement on the quality factor of the cavity is greatly relaxed. The present proposal provides a different approach to quantum computing and communication with superconducting qubits. To the best of our knowledge, there has been no experimental demonstration of entanglement or logical gates for two SQUIDs; and we hope that the proposed approach will stimulate further theoretical and experimental activities.

Before we conclude, we should mention that the idea of coupling multiple qubits and tuning the individual qubits to couple and decouple them from the resonator has been presented previously [9]. Our scheme is much in the same spirit in the sense of coupling and decoupling the individual qubits by manipulation of the Hamiltonians, but it is for a different system and it differs in details of both the qubits and the coupling structure.

\section{ACKNOWLEDGMENTS}

We thank Zhongyuan Zhou and Shi-Biao Zheng for many fruitful discussions and Julio Gea-Banacloche for very useful comments. This work was partially supported by the National Science Foundation (Grant No. EIA-0082499), AFOSR (Grant No. F49620-01-1-0439), funded under the Department of Defense University Research Initiative on Nanotechnology (DURINT) Program, and by the ARDA. 
[1] J.E. Mooij, T.P. Orlando, L. Levitov, L. Tian, C.H. van der Wal, and S. Lloyd, Science 285, 1036 (1999).

[2] C.H. van der Wal, A.C.J. ter Haar, F.K. Wilhelm, R.N. Schouten, C.J.P.M. Harmans, T.P. Orlando, S. Lloyd, and J.E. Mooij, Science 290, 773 (2000).

[3] T.P. Orlando, J.E. Mooij, L. Tian, C.H. van der Wal, L. Levitov, S. Lloyd, and J.J. Mazo, Phys. Rev. B 60, 15398 (1999).

[4] A. Shnirman, G. Schön, and Z. Hermon, Phys. Rev. Lett. 79, 2371 (1997).

[5] A. Blais and A.M. Zagoskin, Phys. Rev. A 61, 042308 (2000).

[6] A. Steinbach, P. Joyez, A. Cottet, D. Esteve, M.H. Devoret, M.E. Huber, and J.M. Martinis, Phys. Rev. Lett. 87, 137003 (2001).

[7] J.M. Martinis and R.L. Kautz, Phys. Rev. Lett. 63, 1507 (1989)

[8] Y. Makhlin, G. Schoen, and A. Shnirman, Rev. Mod. Phys. 73, 357 (2001).

[9] Y. Makhlin, G. Schoen, and A. Shnirman, Nature (London) 398, 305 (1999).

[10] Y. Nakamura, Y. Pashkin, and J.S. Tsai, Nature (London) 398, 786 (1999).

[11] W. Xiang-bin and M. Keiji, Phys. Rev. B 65, 172508 (2002).

[12] W. Xiang-bin and M. Keiji, e-print quant-ph/0105008.

[13] J.R. Friedman, V. Patel, W. Chen, S.K. Tolpygo, and J.E. Lukens, Nature (London) 406, 43 (2000).

[14] X. Zhou, J.L. Habif, M.F. Bocko, and M.J. Feldman, e-print quant-ph/0102090.

[15] Z. Zhou, Shih-I Chu, and S. Han, Phys. Rev. B 66, 054527 (2002).

[16] P. Silvestrini and L. Stodolsky, e-print cond-mat/0004472.

[17] J.I. Cirac and P. Zoller, Phys. Rev. Lett. 74, 4091 (1995).

[18] T. Sleator and H. Weinfurter, Phys. Rev. Lett. 74, 4087 (1995).

[19] M. Brune, S. Haroche, J.M. Raimond, L. Davidovich, and N. Zagury, Phys. Rev. A 45, 5193 (1992).

[20] Q.A. Turchette, C.J. Hood, W. Lange, H. Mabuchi, and H.J. Kimble, Phys. Rev. Lett. 75, 4710 (1995).

[21] M.F. Bocko, A.M. Herr, and M.J. Feldman, IEEE Trans. Appl. Supercond. 7, 3638 (1997).

[22] R. C. Rey-de-Castro, M.F. Bocko, A.M. Herr, C.A. Mancini, and M.J. Feldman, e-print quant-ph/0102089.

[23] M. Crogan, S. Khlebnikov, and G. Sadiek, e-print quant-ph/0105038.

[24] Y. Yu, S. Han, X. Chu, S.-I. Chu, and Z. Wang, Science 296, 889 (2002).

[25] D. Vion, A. Aassime, A. Cottet, P. Joyez, H. Pothier, C. Urbina, D. Esteve, and M. Devoret, Science 296, 886 (2002).
[26] J.M. Kikkawa and D.D. Awschalom, Phys. Rev. Lett. 80, 4313 (1998).

[27] A. Imamoglu, D.D. Awschalom, G. Burkard, D.P. DiVincenzo, D. Loss, M. Sherwin, and A. Small, Phys. Rev. Lett. 83, 4204 (1999).

[28] E. Pazy, E. Biolatti, T. Calarco, I. D’Amico, P. Zanardi, F. Rossi, and P. Zoller, e-print cond-mat/0109337.

[29] M. Bayer and A. Forchel, Phys. Rev. B 65, 041308(R) (2002).

[30] E. Biolatti et al., Phys. Rev. Lett. 85, 5647 (2000); Phys. Rev. B 65, 075306 (2002).

[31] S. Han, R. Rouse, and J.E. Lukens, Phys. Rev. Lett. 76, 3404 (1996).

[32] T.P. Spiller, T.D. Clark, R.J. Prance, and A. Widom, Prog. Low Temp. Phys. 13, 219 (1992).

[33] S.B. Zheng and G.C. Guo, Phys. Rev. Lett. 85, 2392 (2000); Phys. Rev. A 63, 044302 (2001). Zheng and Guo addressed how to realize quantum entanglement and the controlled-NOT logical gate using atoms with a $\Sigma$-type three-level or a twolevel configuration in cavity QED. In our scheme, we instead use the $\Lambda$-type three-level configuration because it is rather difficult to utilize the $\Sigma$-type level structure with SQUIDs.

[34] A. Sørensen and K. Mølmer, Phys. Rev. Lett. 82, 1971 (1999).

[35] J.S. Bell, Physics (Long Island, N.Y.) 1, 195 (1965).

[36] A.K. Ekert, Phys. Rev. Lett. 67, 661 (1991).

[37] D. Deutsch and R. Jozsa, Proc. R. Soc. London, Ser. A 439, 553 (1992).

[38] C.H. Bennett, G. Brassard, C. Crépeau, R. Jozsa, A. Peres, and W.K. Wootters, Phys. Rev. Lett. 70, 1895 (1993).

[39] V. Buzek and M. Hillery, Phys. Rev. A 54, 1844 (1996).

[40] J.W. Pan, M. Daniell, S. Gasparoni, G. Weihs, and A. Zeilinger, Phys. Rev. Lett. 86, 4435 (2001).

[41] C.A. Sackett, D. Kielpinski, B.E. King, C. Langer, V. Meyer, C.J. Myatt, M. Rowe, Q.A. Turchette, W.M. Itano, D.J. Wineland, and C. Monroe, Nature (London) 404, 256 (2000).

[42] E. Hagley, X. Maitre, G. Nogues, C. Wunderlich, M. Brune, J.M. Raimond, and S. Haroche, Phys. Rev. Lett. 79, 1 (1997); S. Osnaghi, P. Bertet, A. Auffeves, P. Maioli, M. Brune, J.M. Raimond, and S. Haroche, ibid. 87, 037902 (2001).

[43] L.X. Li and G.C. Guo, Phys. Rev. A 60, 696 (1999).

[44] M. A. Nielsen and I. L. Chuang, Quantum Computation and Quantum Information (Cambridge University Press, Cambridge, England, 2001).

[45] G. Brassard, S.L. Braunstein, and R. Cleve, Physica D 120, 43 (1998).

[46] M. Brune, E. Hagley, J. Dreyer, X. Maître, A. Maali, C. Wunderlich, J.M. Raimond, and S. Haroche, Phys. Rev. Lett. 77, 4887 (1996). 